Article

\title{
Emergency Service Provision Using a Novel Hybrid SOM-Spiral STC Model for Group Decision Support under Dynamic Uncertainty
}

\author{
Hai Van Pham ${ }^{1, *(1)}$ and Philip Moore 2 (1) \\ 1 School of Information and Communication Technology, Hanoi University of Science and Technology, \\ 1 Dai Co Viet, Le Dai Hanh, Hai Ba Trung, Hanoi 10000, Vietnam \\ 2 School of Information Science and Engineering, Lanzhou University, Feiyun Building, 222 Tianshui S Rd, \\ Chengguan Qu, Lanzhou Shi, Lanzhou 730030, China; ptmbcu@gmail.com \\ * Correspondence: haipv@soict.hust.edu.vn; Tel.: +84-4-823-727555
}

Received: 5 August 2019; Accepted: 11 September 2019; Published: 18 September 2019

\begin{abstract}
In emergency scenarios service vehicles must identify potential route(s) and use the best available route. However, route identification requires intelligent decision-support systems which generally use non-traditional approaches with tools characterised by flexible non-hierarchical structures. Conventional models using group decision-support systems have been applied; however, when used in smart urban environments, emergency response services have limitations in their ability to identify unobstructed paths (routes) in dynamic operating environments. In this paper we introduce a novel path planning method for autonomous vehicle control in emergency situations. The proposed model uses self-organizing maps in an integrated Spiral STC algorithm termed the: Hybrid SOM-Spiral STC model which uses hedge algebras and Kansei evaluation in group decision-support. The proposed model has been designed to quantify qualitative factors using sensor derived data processed with human sensibilities and preferences in emergency decision support. The experimental results show that the proposed model achieves significant improvements in group decision-support under dynamic uncertainty. We posit that our novel approach holds the prospect of improvements in the provision of emergency services.
\end{abstract}

Keywords: self organizing maps; fuzzy rules; emergency planning and management; group decision support; autonomous robot control; smart-environments

\section{Background}

The world has experienced a paradigm shift in the mode of living with a transition from rural living to a growing urban population [1]. In 1800, approximately $3 \%$ of the world's human population lived in urban areas. However, by 1900, the urban proportion rose to approximately $14 \%$ and by 2008 the urban proportion was approximately 50\% [1]. The United Nations predict that on a global basis the urban population will reach $61 \%$ by 2030 and will eventually reach a "dynamic equilibrium of approximately $80 \%$ urban to $20 \%$ rural dwellers that will persist for the foreseeable future" [1]. This transition presents a number of challenges which include many socio-economic, demographic, economic, cultural, and environmental considerations [1].

The literature provides abundant examples of research into these challenges; however, the growth of urban environments presents significant problems for authorities in the effective provision of emergency services. Such services range from the ability of police, fire, and healthcare (e.g., an ambulance) to reach emergencies promptly to the provision of rescue and recovery in disaster (environmental and terrorist) scenarios. A primary concern for authorities tasked with the provision of emergency services is the ability to travel in urban areas efficiently, this is an area that has received 
less attention in the literature. In considering the disasters scenarios human intervention may be impractical or even dangerous. In such cases robotic systems may be employed; rescue robotics are designed to enable emergency responders [and other stakeholders] to "sense and act at a distance from the site of a disaster or extreme incident" [2].

Traversing a dynamic urban environment for emergency service provision demands the identification of potential paths (routes) and the selection of the optimal routes. Such routes will be characterised by a number of factors including: (a) congestion on the road network, to (b) blocked routes in emergency disaster scenarios. In this paper introduce a novel approach which is designed to achieve autonomous emergency service vehicle (or robotic systems) control; the aim is to match the control with emergency response strategies using group decision making implemented in smart dynamic urban environments.

The proposed model utilises a novel path planning method using a self-organizing map (SOM) in an integrated Spiral STC algorithm termed the: Hybrid SOM-Spiral STC (HSSTC) model. The model enables autonomous vehicle control using hedge algebras and Kansei evaluation in group decision-support. The proposed model is designed to quantify qualitative factors using sensor data processed with human sensibilities and preferences [using linguistics and semantics] in emergency scenario decision support. The experimental results show that the proposed model achieves a significant improvement in group decision-support under dynamic uncertainty. We posit that our novel approach holds the prospect of improvements in the provision of emergency services.

The remainder paper is structured as follows: Section 2 provides an overview of the problem with the proposed contribution. A review of related research is set out with a summary in Section 3. We consider situational awareness and pervasive conciousness in Section 4 with smart environments addressed in Section 4.1. The fog computing paradigm is introduced in Section 4.2. In Section 5 we consider materials and methods where we address the problem formation in Section 5.1 with the proposed system set out in Section 6 and emergency strategies quantified using self organising maps introduced in Section 6. Section 9 presents the results with a discussion with consideration of future directions for research. The paper closes with Section 10 where we set out concluding observations.

\section{Problem Statement}

In Section 1 we have introduced the paradigm shift in urban living which has resulted in enlargement of urban centres with vastly increased congestion which is an important element in the provision of efficient and timely emergency response. Emergency response systems require effective route planning (in robotics this may be viewed from the perspective of "path planning" [3-5]) which must implement avoidance of obstacles (static and moving) to achieve the optimal path in traversing the defined operational environment (DOE).

An emergency may be manifested as: (a) a disaster (e.g., an environmental or terrorist) scenario in which an autonomous robot (or an autonomous service vehicle) must navigate the DOE, or (b) an emergency response scenario (e.g., a fire of health emergency) scenario in which emergency response vehicles must be capable of identifying the optimal (i.e., quickest) route to the emergency. Both scenarios require intelligent decision-support systems (DSS) to plan the optimal (or available) routes to the scene of the emergency incident.

For DSS in uncertain environments, the focus must be on knowledge-based decisions using both tacit and explicit knowledge. Decisions are generally made using a 'qualitative approach' to express parameters and preferences [of decision makers] with the aim of determining the optimal decision from a range of alternative options. Decision makers' preferences in emergency and disaster management may be realised using linguistics and semantics (using Kansei evaluation with hedge algebras) in target-oriented decision making in 'real time'.

In this paper we propose an approach designed to quantify qualitative factors using linguistics and semantics with constraint satisfaction (CS) and model human sensibilities in optimal decision making. Our proposed model employs hedge algebras [6] integrated with Kansei evaluation [7] to implement 
autonomous emergency service vehicle management and control with the identification of optimal (even possible) routes to locations in urban disaster zones for service vehicle control. An illustrative example is shown to demonstrate how the proposed model can be controlled car vehicles in emergency. This addresses a disaster recovery and planning for disaster management.

Our contribution lies in the development of an intelligent decision-support system for autonomic path-planning under dynamic uncertainty.

\section{Related Research}

There is a large body of research addressing disaster recovery and planning for disaster management, for example see [8-12].

Comfort in [8] considers risk and security as it relates to disaster management. DSS have presented information and knowledge in many differing formats to stakeholders to enable effective decisions in disaster scenarios [13]. In [12] Comfort addresses crisis management; with hindsight she considers cognition, communication, coordination, and control. Going further, in [10] Comfort investigates complex systems in crisis situations and considers anticipation and resilience in dynamic environments.

Eiser et al. in [9] proposes a conceptual framework for response to natural hazards and considers the framework in the light of risk interpretation and action. Kwan et al. in [11] discusses a practical application of 'real-time' disaster recovery in presenting an analysis of the emergency response following the New York 9/11 disaster. Kwan et al. investigate the potential of real-time disaster.

Jooho et al. [14] have proposed a framework for geographic information systems (GIS) with an investigation into their capability to implement a rapid emergency response in micro-spatial environments. Khan et al. in [15] proposed a warning fire detection system using Convolutional Neural Network (CNN) surveillance for effective disaster management using cameras based on cognitive radio networks to enable reliable data dissemination.

\section{Summary}

In considering the related research, the studies identified are limited in terms of 'real-time' disaster service response. An analysis of the studies clearly identifies the need for a rapid, and importantly, a planned and organised approach to enable effective and timely decisions to be made in 'real-time'. However, all the research identified all approach disaster management and response from a different perspective.

A survey of data management and analysis in disaster situations [16] has identified that disaster management has received increasing attention by computer scientists from multiple research disciplines. The focus of such research has been the drive to devise ways to manage and analyse the data produced in disaster management situations. A limitation in the current methods considered include: (a) they are applied in specific disaster scenarios with no optimal route identification or coordination with control services, (b) the methods have failed to consider or aggregate expert [human] sensibilities and preferences, weather influences, environmental phenomena, and real-time data sets.

In this paper we attempt to address these limitation by introducing our novel approach which implements intelligent autonomous decision-support using hedge algebras integrated with Kansei engineering evaluation. The proposed approach has been conceived to implement autonomous path planning and control of robotic systems applied in emergency scenarios.

\section{Pervasive Conciousness and Smart Environments}

In considering autonomous resources (e.g., robotics and emergency service vehicles) such resources must be aware of their 'world' (the DOE). As such, autonomous resources may be analogous to humans. When navigating their 'world' [17] humans are context-aware [18] and possess pervasive conciousness (PC), this manifests itself in terms of an awareness of their surroundings including the constant (dynamic) change and its affect on their choices and decisions [19]. The goal of intelligent 
autonomic DSS is to model the human ability to perceive their world [the DOE] and act according to the dynamic change; this manifests itself as machine conciousness.

Paul Weiser in the seminal paper [20] argued that: "The most profound technologies are those that disappear. They weave themselves into the fabric of everyday life until they are indistinguishable from it". The vision espoused by Weiser arguably forms the foundation for context-awareness which is generally implemented in pervasive systems; autonomous resource operation will operate in pervasive systems often in smart environments (SE) (we defer consideration of SE to Section 4.1). SE are characterised by networked sensor-based systems which are, by their very nature, invisible to the general public and are therefore pervasive systems.

PC is closely related to situational awareness (SA). In considering the nature of PC and SA, an interesting model of SA is proposed by Endsley in [21]. Endsley and Garland in [22] have observed that SA may be viewed as: "knowing what is going on around you". Going further, Endsley notes that SA (and therefore PC):

"requires a much more advanced level of situation understanding and a projection of future system states in the light of the operator's pertinent goals. As such, situational awareness presents a level of focus that goes beyond traditional information processing approaches in attempting to explain human behaviour in operating complex systems"

While this definition relates to humans, there is a clear correlation with computerised systems [including autonomous path planning for autonomous context-aware DSS] which include machine conciousness. To realise shared goals, agents (both human and computer) must share a common conceptual model [23] which is a shared understanding of the relevant task related processes and goals.

For an excellent discussion on conceptual models as they relate to product design see [24]. Endsley and Garland in [22] refer to such models as mental (or cognitive) models. For computer systems, we may view mental models in terms of computer modelling, however in practice both mental and computer models are types of cognitive model $[18,23,25]$.

$\mathrm{PC}$, as it relates to the topic considered in this paper, addresses the requirement for awareness of the multi-faceted sources of information [which may include raw sensor data and social media sources] that describe the dynamically changing context that exists in 'real-world' environments [21,22]. For example, the monitoring of social media may provide early warning of a major incident to warn the emergency services of the need for implementation of a 'major incident' plan [26].

Autonomous computerised systems (including robotic systems and emergency service vehicles) must possess PC in order to correctly perceive their 'world' (the DOE) which may be a SE as discussed in the following section.

\subsection{Smart Environments}

We have introduced PC which function on the basis of 'relevant' contextual information [27]. The important aspect of contextual information is 'relevance' and the avoidance of 'irrelevant' information which merely increases complexity. $\mathrm{n}$ considering Information Systems (IS) and Information System Design Checkland and Holwell [28] introduced the concept of "CAPTA" which is a concept in which a range of data which will be processed into useful information (or CAPTA).

The CAPTA process introduces an intermediate stage data processing where relevant data is selected and processed into information useful to meet domain specific requirements. We have investigated the relationships between data, relevance, and information as it relates to contextual information in [17]; Figure 1 presents a conceptual model illustrating the progression of data processing from the raw data to wisdom (rational decision-support). Shown is the intermediate stage where an initial selection of relevant and useful raw data (termed CAPTA is used.

In this section we consider the nature of 'smart-environments' which generally refer to two types of environment: (a) 'smart-homes', and (b) 'smart cities'. In this paper the focus is on 'smart-cities'. 


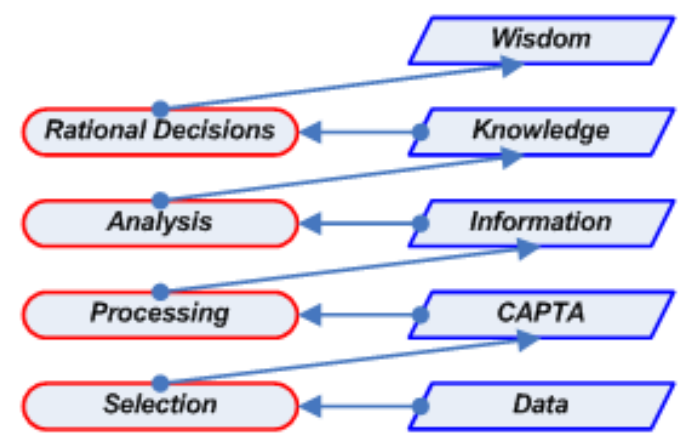

Figure 1. A conceptual model illustrating the progression of data processing from the raw data to wisdom (rational decision-support) (source: [17]).

\section{Smart-Cities}

The concept of a 'smart-city' is difficult to define as there are many interpretations with no common generally agreed definition. Deakin and Al Wear [29] define the smart city as: "one that utilises information and communication technologies (ICT) to meet the demands of the market (i.e., the stakeholders in a city environment)" and "implements ICT to positively impact the local community". From this definition we may conclude that, while a commonly agreed definition remains elusive, the overall goal of improving the environment to the benefit of the [usually urban] population is generally agreed.

What is generally agreed on are the constituent parts that combine to create a 'smart-city', these being: an urban environment, the use and integration of ICT, and the use of networked sensor-based technologies (including the Internet-of-things (IoT) implemented using fog computing technologies) in complex networks to extract raw data for processing. Moreover, a 'smart-city' provides a basis upon which managers may interact with infrastructure systems to monitor, manage and control a broad range of urban systems which may range from traffic management to environmental monitoring and law enforcement. These monitoring functions are implemented in 'real-time' with latent data analytics providing reports with historical information and future projections.

Intelligent autonomous computerised systems as considered in this paper, the goal is to enable (for example a robot or emergency service vehicle to efficiently traverse a DOE by the optimal route. Effective navigation of an urban environment requires the processing of dynamic environmental knowledge. For humans this is generally intuitive, but for machines this requires machine conciousness which may be modelled on human cognitive processes. Achieving this requires the conceptualising of 'smart-environments' with data, information, and knowledge and the related processing in a decision-support system. A feature of a PC is the prevalence of sensors [30,31] which may be considered in terms of the IoT implemented in the fog computing paradigm (also often termed 'edge' computing) [32]. While the terms 'fog' and 'edge' are often used interchangeably, there is a subtle difference as discussed in [32].

\subsection{The Fog Computing Paradigm}

In practice autonomous systems operate on selected sensor derived data processed into information and knowledge [17]. The data will always be generated from a network of sensors including the Internet-of-Things (IoT) and transmitted wirelessly, the data being processed using the fog computing paradigm [32].

Fog computing is closely related to cloud computing [32,33]. Cloud computing is a multidisciplinary field which features the convergence of several independent computing technologies and trends including: Internet and internet delivery, pay-as-you-go services, utility computing, elasticity, virtualisation, grid computing, distributed computing, storage, content outsourcing, security, and Web 2.0/Web 3.0 [34]. 
The multidisciplinary nature of cloud computing has raised many open research questions due to the modes(s) of implementation given that the cloud computing paradigm incorporates "almost everything that existing technologies already do" [34]. However, the cloud computing paradigm was "not designed for very large volume, variety, and velocity of data generated by the IoT" [32]. Accordingly, there are significant issues related to latency [35] and "by the time the data reaches our cloud for analysis, the opportunity to make decisions may be lost" [32].

This issue represents a significant problem for 'real-time' data processing in DSS, low-latency 'real-time' data processing requiring the processing of data close to the source of the data [35]. The investigation of fog computing paradigm provides a basis upon which this may be realised; for a detailed discussion see [35]. In considering the focus of this paper, the fog computing paradigm as expressed in our fog computing model provides an effective controlled latency approach to provision of low-latency, task specific, 'real-time' data processing in an emergency scenario.

\section{Materials and Methods}

We have considered the background to the study; we now turn to the problem formulation, consider Kansei words and hedge algebras with the matrix construction for an emergency environment, consider the Suffix Tree Clustering (STC) algorithm, and introduce the proposed integrated Spiral STC algorithm termed the: HSSTC algorithm.

\subsection{Problem Formulation and Matrix Construction}

The principal issue in emergency response are the identification of the optimal path(s) (or route(s) to the location of the emergency; in extreme emergencies the issue may extend to identifying available paths. Emergency response management centres must be aware of the DOE and the current situation relating to emergency service resources. Additionally, emergency management centres must apply strategic management to manage dynamic 'real-time' emergency situations; such strategies will involve the deployment of resources and the optimal routing of the resources matching the resources to the prevailing emergency as it develops 'on-the-ground'.

We may formulate a conceptual emergency domain as follows:

1. The Hedge-Emergency-DSS: the objective function is designed to maximise the operational efficiency of the emergency management decision making when dealing with multiple emergencies;

2. Let $X^{S}=\left\{X_{1}^{S}, X_{2}^{S}, \ldots, X_{m}^{S}\right\}$ be a set of adjectives called Kansei words to consider available alternatives in evaluating an emergency situation where $m$ is the number of Kansei words;

3. Assume that quantification of human sensibilities using in decision making can be presented in an emergency situation domain $S$; Optimized Kansei words in $X^{S}$ are used to evaluate alternatives which belong to the criteria and factors $S$;

4. Let $W_{m}^{S}=\left\{W_{m}^{-}, W_{m}^{+}\right\}$be adjective pairs of Kansei words belonging to $X_{m}^{S}$;

5. Let $P$ be decision makers' input the proposed system in $S$;

6. In evaluation of alternative $X_{j}^{S}$, its Kansei weight $w_{i j}^{t}$ can be represented by the $i$-th Kansei word of the $j$-th alternative considered by the $t$-th decision maker.

7. Let $V^{S}=\left\{V_{1}^{S}, V_{2}^{S}, \ldots, V_{h}^{S}\right\}$ be a set of transportation vehicles $S$, where $h$ is the number of emergency vehicles.

8. Let quantitative semantics work in the mapping of $(H A)$, presented in linguistic values for $\left(w_{j}\right)$ fall in the range $[0,1]$.

9. Let a decision maker calculate an objective function value for $\left(Q_{j}(X)\right)$ in the emergency domain

10. Group Decision Making (GDM) is a solution for two or more decision makers to aggregate their preferences. Let $E^{S}=\left\{e_{1}^{S}, e_{2}^{S}, \ldots, e_{q}^{S}\right\}$ be a set of experts for a selection of disaster strategies, where $q$ is the number of experts. Let $G^{S}=\left\{g_{1}^{S}, g_{2}^{S}, \ldots, g_{c}^{S}\right\}$ be a set of expert groups, where $c$ is the number of expert groups. Let $p_{t}^{S} \mid(t=1, \ldots, c)$ represent disaster strategies determined by an expert $e_{j}^{S}$.

11. In an emergency domain $S$, each expert may determine an approach that matches the appropriate emergency management strategies based on the results of Kansei evaluation. 
12. Tor identify disaster strategies $p_{t}^{S}$, an expert $e_{i}^{S}$ provides his/her preference in a five-point scale definition (0: oppose, 0.25: almost oppose, 0.5: have no preference, 0.75: almost agree, 1: agree) to evaluate companies. We construct a Decision matrix $A_{q \times k}^{S}=\left(a_{i j}^{S}\right)_{(q \times k)}$, where $k=l+m+g$ is the total number of Kansei words, disaster quantitative and qualitative factors, and $q$ is the number of experts.

\subsection{Kansei words and Hedge Algebras in an Emergency Environment}

In this section we address the use of Kansei words with hedge algebras and the Kansei emergency matrix. Consider 'real-world' emergency response: when decision makers evaluate alternative approaches (which are qualitative in nature) the evaluation is generally expressed in linguistic (semantic) terms such as: "satisfied", "acceptable", or "good". These methodologies have been shown to solve the challenges in the manipulation of linguistic information in decision-making problems. However, these studies have failed to determined adjective pairs such as: Synonym-Antonym and Synonym-Not Synonym. For example, pairs of adjectives such as: good-bad and successful-unsuccessful are essential when used in decision making under uncertain dynamic environments. Research has identified situations in disaster responses where quantification of human sensibilities and perceptions together with decision supports is systemic requirement.

Decision makers' preferences on Kansei attributes in target-oriented decision analysis provides a good description of individual preferences. Additionally, the target-oriented decision analysis has been used to quantify factors in terms of evaluating alternatives. Kansei evaluation (KE) [7] is a systematic determination of the significance of decision makers' preferences when measured using criteria and measured against a set of Kansei attributes. Kansei evaluation is a commonly used method for evaluation to enable quantification of impressions. Kansei is given by adjectives describing human emotion, sensibility and impression [7].

Representations of a linguistic valued qualitative aggregation and human reasoning for multi-criteria decision making is useful when applied in DSS; for example see: Chen et al. [36] and Liu et al. [37]. These studies have investigated linguistic valued logic based approximate reasoning methods for use in DSS; Chen et al. [36] and Liu et al. [37] have reported success in resolving challenges and problems in perception-based decision making.

The approach presented in this paper proposes an approach which uses hedge algebra [6] integrated with Kansei evaluation [7] to enable context-matching [27,38] and address the limitations identified in Chen et al. [36] and Liu et al. [37]. Linguistic information involved in multi-criteria decision problems in an emergency domain with a logic-based approximate reasoning method has been developed by Chen et al. [36] to provide decision-support based on information provided.

To evaluate potential factors and alternatives (inputs) and Kansei words): we have used the range $[0, \ldots, 1]$ which is poset of a Hedge algebra. For example, we can consider the set of all possible truth values: where $\mathrm{T}=$ true, false, very true, very false, approximately true, possibly true, approximately true or possibly true, approximately true and possibly true,... These scales are used in the logic reasoning based decision making that is capable of handling both totally ordered and partially ordered linguistic information directly without numerical approximation.

The purpose of Kansei emergency matrix construction is to quantify Kansei, emergency (disaster) data sets, together with expert preferences for emergency response, represented as fuzzy weight values $[0,1]$. The matrix is visualized by a SOM for training to find emergency vehicles, together with expert's sensibilities and preferences applied in matching resource allocation and path planning with appropriate emergency management strategies.

\subsection{The Spiral STC Algorithm in an Emergency Environment}

In the Spiral STC (STC) on-line algorithm, a vehicle applies using coverage path algorithm relating to information in an emergency domain. 


\section{STC on-line Algorithm:}

Sensors: Vehicle position and direction sensors, detect using sensors.

Input: Location of start mega-cell $S$ and no information about the environment.

Recursive function: STC $(\mathrm{w}, \mathrm{x})$, where $\mathrm{x}$ is the current node, $\mathrm{w}$ is the parent node in spanning tree. Initialization: STC (null, S).

\section{Algorithm STC:} Spiral-STC $(w, x)$ :

1. Mark the current a root cell $x$ as "visited"

2. While x still has an "unvisited" adjacent that is not completely occupied by an obstacle or vehicle.

2.1. Search for new neighbours of $\mathrm{x}$ in an anti-clockwise direction. Call $\mathrm{y}$ the first neighbor of $x$.

2.2. Build a connection edge between $x$ and $y$.

2.3. If $y$ is completely free:

From $x$ move to a sub-cell of $y$ along the output edge of $x$.

2.4. If $\mathrm{y}$ is partially occupied by an obstacle or vehicle:

\subsubsection{Build C-Space space at $\mathrm{y}$.}

2.4.2. From $x$ just moving along the new contour of $C$-Space at $y$ has just followed the access edge of $y$ to return to $x$.

2.5. Call STC $(x, y)$

3. End the loop.

4. If $x$ differs from $S$, move back to $x$.

5. Returns results $(\mathrm{STC}(\mathrm{w}, \mathrm{x})$ stops).

\section{The Proposed System}

In uncertain (dynamic) emergency environments, disaster response decisions are by their very nature very urgent. A time consuming simulation process to search for optimal solution(s) for service vehicles supporting an developing emergency is neither realistic or desirable. A robust IT communication infrastructure of vehicles in will enhance safety and provide information in 'real-time' which can result in a quick response time to potentially fatal situations. A conceptual view of the proposed system to implement emergency response is shown in Figure 2.

Qualitative decision making under uncertain environments, while including explicit and tacit knowledge [39], is also significantly impacted by sensor signals, experience, and human sensibilities. In such circumstances, decision making may be transformed by the use of approximate reasoning.

Human decisions are driven by reasoning and inference; natural language giving meaning and context to the factors that combine to inform decisions. Identifying linguistically (semantically) and evaluating the factors to be considered in reaching a decision or conclusion provides a basis upon which emergency situations may be (at least partly) controlled. For example, in the case of a disaster emergency [in a disaster zone] the the optimal method for the human may be identified from range of potential alternatives. In reaching such decisions, quantifying human reasoning, perception, and linguistic (semantic) information performs a significant role in decision making under uncertain disaster environments. 


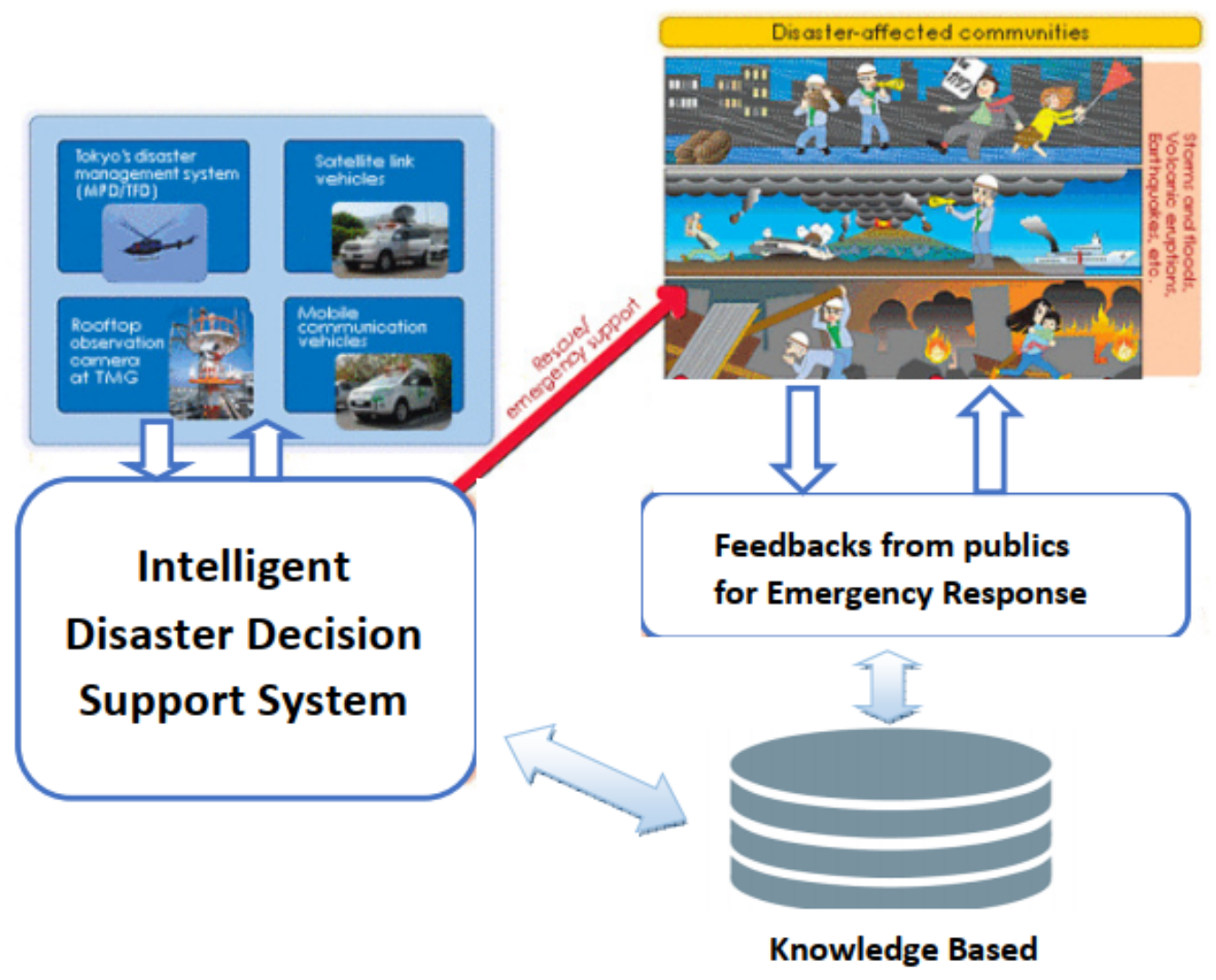

Figure 2. Overview of the proposed system using the Internet-of-Things (IoT). A conceptual view of the proposed system to implement emergency response.

For humans, language and human sensibility in the decision-making process for emergency response situations in particular] where language and communication form an essential component; Kansei evaluation represents human sensibilities taken together with decision making in 'ranking' of final decisions. In natural language, human reasoning can be presented in linguistic (semantic) terms to describe alternatives based on the contextual properties [6]. The proposed system describing (pre emergency, an actual emergency, and post emergency scenarios) is shown in Figure 3.

Figure 3 presents a conceptual model of our proposed system and shows the components that combine to implement our proposed method. The system components that create our proposed decision-support system are introduced below in points 1 to 5 :

1. The Application Interface: provides an effective basis upon which decision makers can reach decisions based on expert preferences.

2. A SOM: is a software tool designed to implement an unsupervised learning algorithm. Developed by Teuvo Kohonen [40], a SOM is a computational method to achieve an orderly mapping of a high-dimensional distribution onto a regular low-dimensional grid, for a detailed exposition on SOM's see [40]. The results from a SOM are the ability to convert complex non-linear statistical relationships between high-dimensional data into simple geometric relationships in a low-dimensional display [40]. Given that a SOM compresses information while retaining the most important "topological and metric relationships of the primary data items on the display" in can also be considered in terms of "some kind of abstractions" [40]. The two results for a SOM (visualisation and abstraction) may be used in a number of ways in complex tasks such as "process, analysis, machine perception, control, and communication" [40].

3. A SOM is usually constructed using a two-dimensional (regular) grid of nodes; Figure 3 shows the use of the Kohonen SOM in the context of our proposed model. Our proposed model has been 
modified to utilise clustering based on a Kohonen SOM to quantify qualitative and quantitative factors together with collaborative decision preferences and sensibilities.

4. DSS Database: The DSS database as knowledge based in the proposed model consists of sensor data, Kansei data set, which responds to decision makers based on their preference in dynamic disaster environments. Most processes of training data sets and fuzzy rules in reasoning are executed in the DSS database.

5. Disaster map: The proposed model is embedded with GIS map using Google map connected through API. The vehicles in tracking GPS via GIS map are identified by the locations on the map.

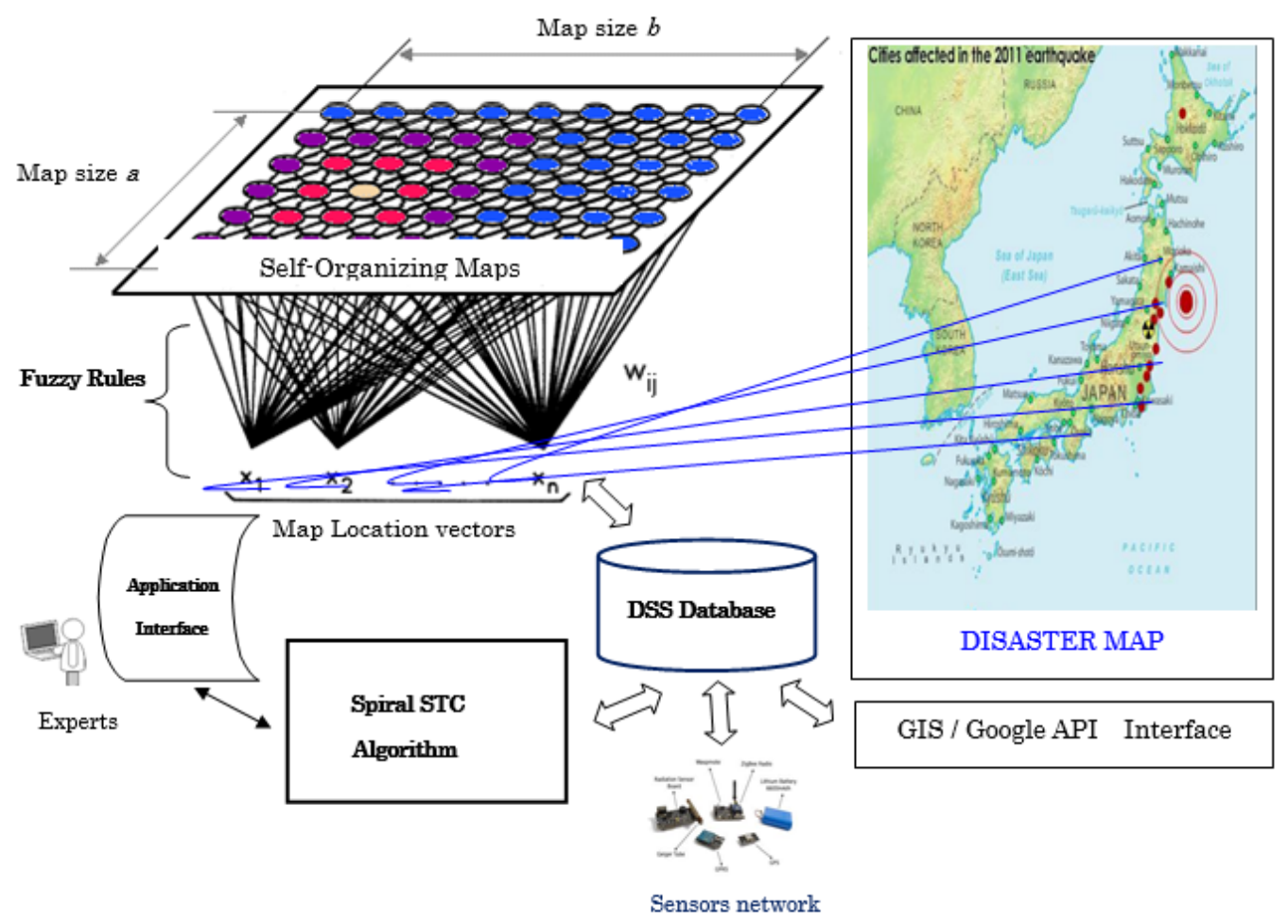

Figure 3. The proposed system showing pre emergency, an actual emergency, and post emergency scenarios.

Emergency Strategies Quantified by Training Self-Organising-Maps

Step 1. Input data sets getting from emergency domain sensors and expert preferences for matching disaster strategies located points in a map: let a decision maker belonging to $E^{S}=\left\{e_{1}^{S}, e_{2}^{S}, \ldots, e_{q}^{S}\right\}$ be a set of experts for a selection of emergency strategies, where $q$ is the number of experts that select one or several emergency strategies $Q_{t}^{S} \mid(t=1, \ldots, c)$ with its data normalization in fuzzy weights in the range $[0,1]$ and his/her preference $\beta_{j}^{S}$ can be defined in a five-point scale (0: oppose, 0.25 : almost oppose, 0.5: have no preference, 0.75: almost agree, 1: agree).

Step 2. Construction of Kansei emergency matrix: Kansei emergency matrix $M_{n \times p}^{S}$ is constructed using fuzzy weight values in the range $[0,1]$ for the results of the Kansei evaluation and the fuzzy evaluation model for emergency strategies as described in Section 5.1. Let $V^{S}=\left\{V_{1}^{S}, V_{2}^{S}, \ldots, V_{h}^{S}\right\}$ be a set of emergency resources (vehicles) in the Kansei disaster matrix $M_{n \times p}^{S}$ in an emergency domain $S$, where $h$ is the number of emergency resources (vehicles) with rules considered by experts and data set(s) are expressed and updated in the DSS database.

Step 3. Visualisation of Kansei disaster matrix by SOM: to find either emergency strategy matching with expert preferences or emergency domains. 
Step 3.1. Definition of Kansei disaster distance: in the training process to evaluate differences among Kansei emergency attributes of emergency vectors by a SOM, the Kansei emergency attribute distance $d_{C_{i} \rightarrow C_{j}}^{S}$ between two vectors $D_{C_{i}}^{S}$ and $d_{C_{j}}^{S}$ represents attributes of emergency vectors $C_{i}^{S}$ and $C_{j}^{S}$ respectively as defined by Euclidean distance given by Equation (1):

$$
d_{C_{i} \rightarrow C_{j}}^{S}=\left\|D_{C_{i}}^{S}-D_{C_{j}}^{S}\right\|
$$

Step 3.2. Visualising the location emergency matrix by a SOM: the location emergency matrix $L_{x \times k}^{S}$ is visualised by a SOM in order to find the similar attributes of emergency vectors matching with emergency locations based on the SOM map.

Step 3.3. Visualising Kansei disaster matrix by the SOM: the Kansei disaster matrix $D_{n \times p}^{S}$ is visualized by a SOM in order to find emergency management strategy matching with expert preferences. To calculate the weights of decision maker preference distances together with a selected emergency strategy $p_{t}^{S} \mid(t=1, \ldots, c)$, the decision maker preference distance $d_{e_{i} \rightarrow e_{j}}^{S}$ is represented by $m_{i j}^{t}$ calculated from the Kansei emergency attribute distance $v_{i j}^{t}$ evaluated by a decision maker $e_{i}^{S}$ of his/her group at iteration $t$ and the Kansei disaster weight $w_{i j}^{t}$ of the decision maker group. The weight of $m_{i j}^{t}$ is expressed by Equation (2):

$$
m_{i j}^{t}=\left\|\frac{1}{P} \sum_{j=1}^{k} w_{i j}^{t}-v_{i j}^{t}\right\|
$$

where $(i=1, \ldots, q, j=1, \ldots, k)$ and $p$ is the number of decision makers in each group.

Step 3.4. Updating Kansei disaster weights: a Decision matrix $D_{q \times k}^{S}$ is updated by its weights given by Equation (3). Then, the Decision matrix $D_{q \times k}^{S}$ is combined with the Kansei emergency matrix $M_{n \times p}^{S}$ and its weights are updated in the matrix as shown in Equation (3)

$$
m_{i j}^{t+1}=m_{i j}^{t}+\beta_{j}^{S}\left(\left\|\frac{1}{P} \sum_{j=1}^{m} w_{i j}^{t}-v_{i j}^{t}\right\|\right)
$$

where $\beta_{j}^{S}$ is a set of decision maker preferences as defined in a five-point scale (0: oppose, 0.25 : almost oppose, 0.5: have no preference, 0.75: almost agree, 1: agree). To select $c$ emergency strategies $\left(p_{1}^{S}, p_{2}^{S}, \ldots, p_{c}^{S}\right)$, steps 3.1 to 3.4 are repeated until $c$ decision maker groups with updated weights in the emergency matrix are complete. The appropriate emergency strategies are matched with decision maker preferences.

Step 4. Selection of vehicles matching with disaster strategies: following the updating of the weights in the emergency matrix of Kansei matrix $M_{n \times p}^{S}$, in Step 4.2 the Kansei matrix $M_{n \times p}^{S}$ is continuously visualised by the SOM. The process from Step 3.2 to Step 4.2 is and repeated until the compromised and aggregated expert preferences in closed grouping points. Based on Kansei emergency distance among expert preferences with the closest emergency resource (vehicle) having similar attributes, the final result on a SOM is shown in the selected emergency resources (vehicles) $V^{S *}=\left\{V_{1}^{S}, V_{2}^{S}, \ldots, V_{h}^{S}\right\}$ in emergency domain $S$ where $h$ is the number of vehicles matched with appropriate emergency strategies.

Step 5. Select priority of vehicle routes in disaster environments: quantification of algebraic linguistics is an interpretation of an algebra. In the formula: $A X=(X ; G ; H ; \leq)$ where $(X ; \leq)$ is a poset. Let $(G)$ be a set of the primary generators and $(H)$ be a set of unary operations that present in terms of linguistic hedges [6]. Linguistic variables can be presented in values Truth such as: True, VeryTrue; alternatively, values False can be presented such as: Probably False, Mostly False, Very Probably False, Probably False and so on. The values given in a set of generators can be defined as follows: 
$(G)=\{$ False , True $\}$ using hedges belonging a set $\mathrm{H}=\{$ Very , Much, More, Truly, Probably, ... $\}$ as unary terms.

The steps in mainly process of the proposed model is depicted as follows:

Step 5.1. Identify the objectives of multiple decision making for emergency for support vehicles: the proposed model for the Hedge-Emergency-DSS is proposed while identifying multiple objectives for the optimal paths in priority selection of emergency service vehicles as shown in Equation (4):

$$
H D D=\frac{\operatorname{Min}}{x} \sum w_{j} \cdot Q_{j}(X)
$$

where the constraints include:

1. $\sum_{k} X_{i k}=1 \forall i \in N$

2. $\sum_{i \in V_{j} U V_{i}} x_{i 1} \geq 1$

3. $x_{i k}=\left\{\frac{1, \text { If option } i \text { is assigned avehicle to mission }(k)}{0, \text { other }}\right.$

Step 5.2. Identify an objective function dealing with multiple decision-making objectives: $\left(Q_{j}(X)\right)$ can be presented as a linguistic value in the variables for quantitative semantics depicted in $(H A)$ and these linguistic values in the fuzzy weights [0.1];

Step 5.2.1. the decision variables $\left(X_{i j}\right)$ can be defined in multiple criteria decision-making objectives for the emergency domain;

Step 5.2.2. the Input human preferences together with Kansei words (Synonym-Not Synonym, Synonym-Antonym): Kansei words in $X^{S}$ or another word called Adjectives are used to evaluate an alternative belonging to criteria in domain $S$. Let $W_{m}^{S}=\left\{W_{m}^{-}, W_{m}^{+}\right\}$be Kansei words or pairs of adjectives involved in decision making in alternatives $X_{m}^{S}$.

Step 5.3. Evaluate the context match in optimal objective decisions: to use Kansei words and Hedge algebras in alinguistic variable Truth such as True, Very almost True, Probably Very False, Very Probably False.

Step 5.4. Mean joining in multiple decision-making processes for the emergency vehicle tasks: $\left(V_{i}^{S}\right)$ and where $\left(\left(V_{i}^{S} \cap V_{j}^{S}\right)=(\theta, \forall i, j \in\{1,2, \cdots r\})\right)$;

Step 5.5. Find either the shortest way or coverage path planning: to find the shortest path (route), a decision maker can apply Dijkstra's algorithm for a vehicle to find the best route to an emergency destination [for a vehicle] using the disaster map. To cover all paths identified in monitoring emergency locations a decision maker can apply the STC algorithm to coverage path in disaster areas shown on a map.

Step 5.6. Consider rule and update its weight to DSS database: for each node of $\left(V_{i}^{S}\right)$ visited, consider Rule $(j)$ represented by $\left(R_{j}\right)$ and apply $\left(V_{i}^{S}\right)$ as follows. In fuzzy rule-based reasoning, the fuzzy rules are generally expressed for two input variables and one output as a decision variable, as given by Equation (5):

$$
\left\{\begin{array}{l}
R^{i}: \text { if } x_{1} \text { is Condition } A_{i 1} \text { and } x_{2} \text { is } A_{i 2} \text { then } y \text { is } V_{i} \\
\vdots \\
R^{n}: \text { if } x_{1} \text { is Condition } A_{n 1} \text { and } x_{2} \text { is } A_{n 2} \text { then } y \text { is } V_{n}
\end{array}\right.
$$

where $x_{1}$ and $x_{2}$ represent input-quantitative variables $\left(x_{1} \in X_{1}, x_{2} \in X_{2}\right)$ to a fuzzy system and $y$ represents output-decision variable $(y \in Y) . A_{i 1}, A_{i 2}$ and $B_{i}$ are fuzzy subsets of $X_{1}, X_{2}$ and $Y$, 
respectively. When the non-fuzzy input data " $x_{1}$ is $x_{1}^{*}$ and $x_{2}$ is $x_{2}^{* \prime}$ is given, the matched context degree $\left(w_{j}\right)$ is calculated by the weight $\left(w_{j}\right)$ : representative of $\left(V_{i}^{S}\right)$ and $\left(w_{j}\right)$.

Step 5.7. Find the appropriate rules considered by experts and applied these weights and rules in Knowledge Base (KB): IF a checking rule is existent in the KB THEN do an action in the considered rule ELSE a new rule matched in the proposed model that can be added to the KB.

All repeated steps are complete and the vehicle path is identified with its multiple decision-making objectives complete the action(s). The final group decision making is to show ranking list $V_{i}>V_{j}>$ $V_{k}>V_{t}>V_{p} \ldots$ for a priority vehicle in the optimal route for emergency response.

\section{Evaluation}

In this section we set out a relevant example with experimental testing to evaluate the proposed approach using a case study predicated on an emergency response scenario.

\section{Example Illustration}

An illustrated example is presented to show how Input data sets obtained from the DOE sensors and expert preferences are used in the matching of emergency strategies with located emergency locations on a map. Let decision maker belonging to $E^{S}=\left\{e_{1}^{S}, e_{2}^{S}, \ldots, e_{5}^{S}\right\}$ be a set of experts for a selection of emergency strategies where $q=5$ is the number of experts that select one or several emergency strategies $Q_{t}^{S} \mid(t=1, \ldots, 5)$ with data normalization in fuzzy weights $[0,1]$ and his/her preference $\beta_{j}^{S}$ defined in a five-point scale (0: oppose, 0.25: almost oppose, 0.5: have no preference, 0.75: almost agree, 1: agree).

In many decision problems related to the prioritisation of emergency service vehicles in emergency response scenarios an emergency service resource (i.e., a robot or vehicle) will identify potential targets, locations, and tasks; this requires multiple decision-making objectives. $\left(Q_{j}(X)\right)$ is an objective function for five disaster strategies indicated for multiple objectives updated in the decision maker's knowledge-base. In steps 1 to 4 [of the proposed system-see Section 6] a decision maker can instruct an on-line emergency DSS on the automatic selection of the emergency management strategies in multiple decision-making objectives as follows: $V_{1}$ (Ambulance), $V_{2}$ (helicopter), $V_{3}$ (fire engine), $V_{4}$ (police car), and $V_{5}$ (rapid fire truck).

Assume that the objective function aims to minimise the impact of a fire in 'real-time' emergency response to meet the five disaster strategies following objectives:

1. $(Q(1))$ : To rescue people in advance of a fire;

2. $(Q(2))$ : To rescue people and move items to a place of safety;

3. $(Q(3))$ : To protect items and infrastructure and maintain safety;

4. $(Q(4))$ : To move items into safe storage;

5. $(Q(5))$ : To rescue people in medical centres and hospitals together with emergency support.

For instance, the value $\left(Q_{j}(X)\right)$ is considered by using the linguistic variables as follows: very high, high, very very low, very little low, medium high, very low, roundly high, ... In the preliminary experiment as shown in Step 5 [steps 5.1-5.7 of the proposed system-see Section 6] Kansei evaluation models human sensibilities in the decision-support function. We have collected 10 Kansei words belonging to an objective function as shown in in Table 1.

A Kansei disaster matrix is constructed using Kansei words as shown in in Table 2.

In Kansei evaluation we us a semantic differential (SD) method with a five-point scale definition: (0: little low, 0.25: low, 0.5: little high, 0.75: high, 1: very high). The values for the preferences of decision makers to evaluate the objective function is depicted in Table 3. From steps in the calculations as discussed above, the degree of the objective function corresponding to the emergency rescue can be shown in Table 4. 
Table 1. Adjectives-Kansei words in Kansei evaluation.

\begin{tabular}{cccc}
\hline No. & Positive Word & Negative Word & Criteria \\
\hline 1 & Dangerous & Not Dangerous & \\
2 & Important & Not important & $Q_{1}$ \\
3 & Serious & Not serious & $Q_{2}$ \\
4 & safety & Unsafely & $Q_{3}$ \\
5 & Successful & Unsuccessful & \\
6 & Risky & Not risky & $Q_{4}$ \\
7 & Satisfactory & Unsatisfactory & \\
8 & Complicated & Not complicated & $Q_{1}$ \\
9 & Certain & Uncertain & \\
10 & Failed & Not failed & $Q_{5}$ \\
\hline
\end{tabular}

Table 2. Adjectives for Kansei evaluation with its criteria evaluation.

\begin{tabular}{cccc}
\hline No. & Positive Word & Negative Word & Criteria Evaluation \\
\hline 1 & Dangerous & Not Dangerous & Location \\
2 & Important & Not important & Emergency \\
3 & Serious & Not serious & Rescue \\
4 & safety & Unsafely & Assessments \\
5 & Unsuccessful & Successful & Support \\
6 & Risky & Not risky & Disaster response \\
7 & Satisfactory & Unsatisfactory & Cost \\
8 & Complicated & Not complicated & Difficulty \\
9 & Certain & Uncertain & Confidence \\
10 & Failed & Not failed & Technology \\
\hline
\end{tabular}

Table 3. Scales in sensibilities of decision makers on the objective function.

\begin{tabular}{ccccc}
\hline & $Q_{1}$ & $Q_{2}$ & $Q_{3}$ & $Q_{4}$ \\
\hline$V_{1}$ & very little high & little very low & very low & little low \\
$V_{2}$ & little low & very low & very high & low \\
$V_{3}$ & low & little low & very high & very low \\
$V_{4}$ & very high & little high & little low & little little low \\
$V_{5}$ & little very high & very little high & little high & very little high \\
\hline
\end{tabular}

Table 4. The objective function weights with the degree of disaster rescue.

\begin{tabular}{ccccc}
\hline & $\boldsymbol{W}_{\mathbf{1}}$ & $\boldsymbol{W}_{\mathbf{2}}$ & $\boldsymbol{W}_{\mathbf{4}}$ & $\boldsymbol{W}_{\mathbf{4}}$ \\
\hline$V_{1}$ & 0.61 & 0.61 & 0.26 & 0.835225 \\
$V_{2}$ & 0.26 & 0.26 & 0.61 & 0.26 \\
$V_{3}$ & 0.26 & 0.26 & 0.26 & 0.26 \\
$V_{4}$ & 0.5365 & 0.5365 & 0.169 & 0.5365 \\
$V_{5}$ & 0.5365 & 0.5365 & 0.7465 & 0.15225 \\
\hline
\end{tabular}

Apply the STC algorithm as shown in Equation (6):

$$
\frac{\operatorname{Min}}{x} \sum_{j} w_{j} \cdot Q_{j}(X)=\operatorname{Min}[0.86756,0.6525,0.3087,0.9687,0.6876]=0.3087
$$

In the proposed Hedge-Emergency-DSS model, multiple decision-making processes [for service resources (vehicles)] will find the optimal path (route) in the emergency zone (the DOE) based on vehicle priorities. As calculated by the formulas above, the illustration example the order of vehicles is expressed by: $\left(V_{4}>V_{1}>V_{5}>V_{2}>V_{3}\right)$. The relevant example demonstrates that vehicles can find the optimal path (route) while maximising the coverage (of the DOE) and monitoring the priority of the emergency service vehicles in emergency response. 


\section{Experimental Results}

The experiments have been implemented using Java, the maps are rendered using the JXMapViewer library embedded with Google maps using an API. The disaster zone can viewed using a Smart phone or desktop application, the view showing the location and extent of the disaster zone along with the location of the emergency service vehicles.

\subsection{Emergency Case study in Vietnam}

To begin the evaluation of the proposed approach to group decision making using the Google maps we conducted an experiment based on a 'real-world' emergency in Vietnam. In this experiment, experts using smart phones or smart devices are embedded in vehicles loaded Kansei data sets for the proposed system. The Kansei matrix was constructed with dynamic dimensions [Kansei words, quantitative, and qualitative factors] represent vehicles from the DSS database of the Vietnam-Japan project disaster centre. In the proposed system, the SOM model uses a Gaussian neighborhood function with an adaptive variance and learning rate.

The parameters are set in the Kansei SOM application $(\mathrm{SOM}$ sizes $=30 \times 30$, Sigma max $=12$, Sigma $\min =3$, Iteration set $=30$ and learning rates from 0.05 to 0.01 ). To consider decision maker preferences in the proposed system, the experts were divided into three groups based on emergency management strategies as objectives in group decision making. The proposed system describing pre-emergency situation, the situation during the emergency, and the post-disaster situation is shown in Figure 4.

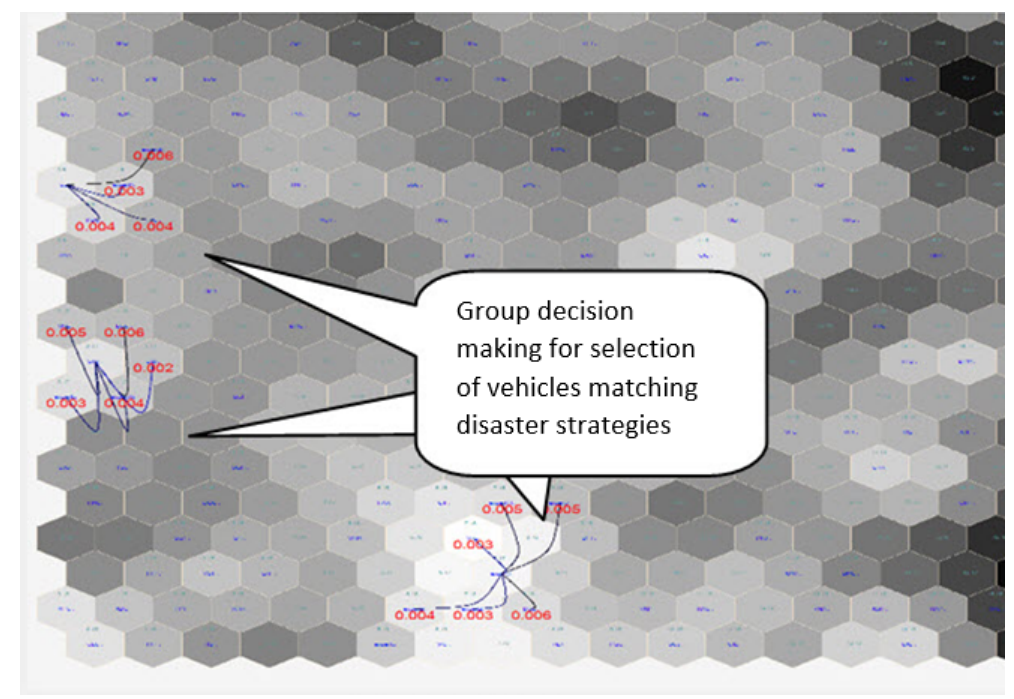

Figure 4. The SOM map results in matching emergency strategies for emergency case study in Japan.

In simulations, the map result showed the Kansei distance of potential emergency response vehicles from the location of the emergency. As observed from Figure 5, decision makers selected these vehicles, matched with appropriate emergency management strategies, by reducing the maximum Kansei disaster distance of the disaster vectors and eliminating those that have a greater distance. Figure 5 illustrates potential vehicles going to the route in disaster domain, aggregated by expert preferences on the map.

In this case study, a human agent (the decision maker) can freely select a strategy [from a range of suggested strategies] in a disaster response scenario. For example, in disaster strategy (1), the decision maker can select the most appropriate strategy to maximise the response (the rescue of individuals using a range of potential implementation options which may include: ambulances, emergency vehicles such as police and emergency service vehicles, fire engines, and helicopters) in a fire disaster situation. 
However, in considering disaster strategy (2) (as shown in Figure 5), the identification and selection of the optimal strategy to maximise the rescue of individuals trapped in a fire must consider current 'real-time' traffic congestion within a city and the optimal solution must select the quickest route (which may not be the shortest route). For instance, in the case of disaster 3 the optimal solution [as identified by a number of experts] has been achieved by implementing control limited to ambulances and fire engines deployed to react to the disaster.

To identify warnings and emergency response notifications, decision maker(s) can input preferences with single or multiple locations and objectives. Let us assume that the emergency service vehicles are shown on Google map belonging to the disaster strategy (2) as shown in Figure 5.

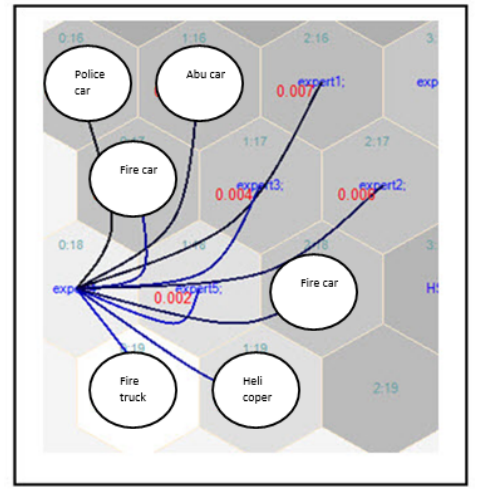

Disaster strategy 1

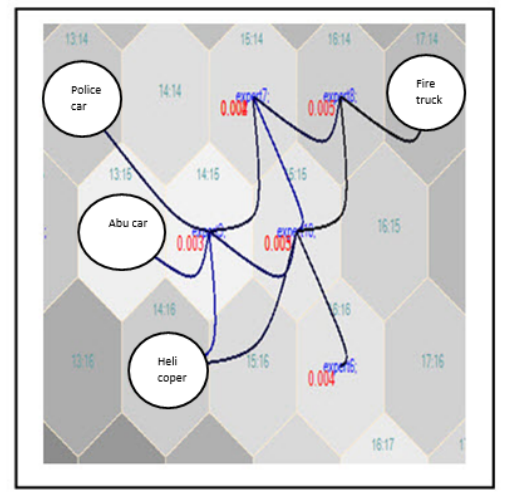

Disaster strategy 2

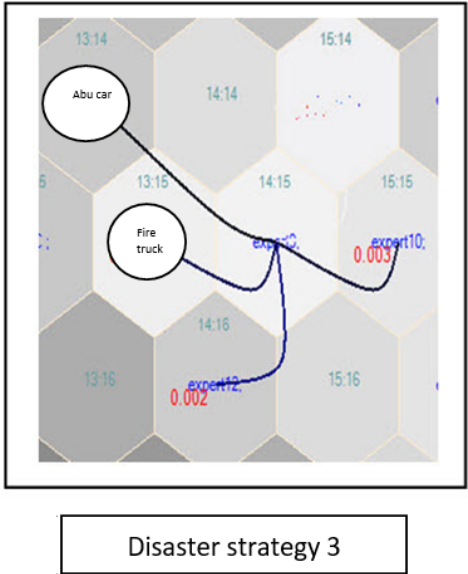

Disaster strategy 3

Figure 5. The SOM map results in viewed vehicles in emergency strategies.

Following entering the decision maker(s) preferences, the experimental results are shown on the map by the combination of transportation and priority. $\mathrm{He} /$ she can find the optimal route(s) while the vehicles moved to the location of the fire in 'real-time'. In the process of multiple decision-making, decision makers can consult a simulation of the vehicles and their movement with locations on the Google map, as shown in Figure 6.

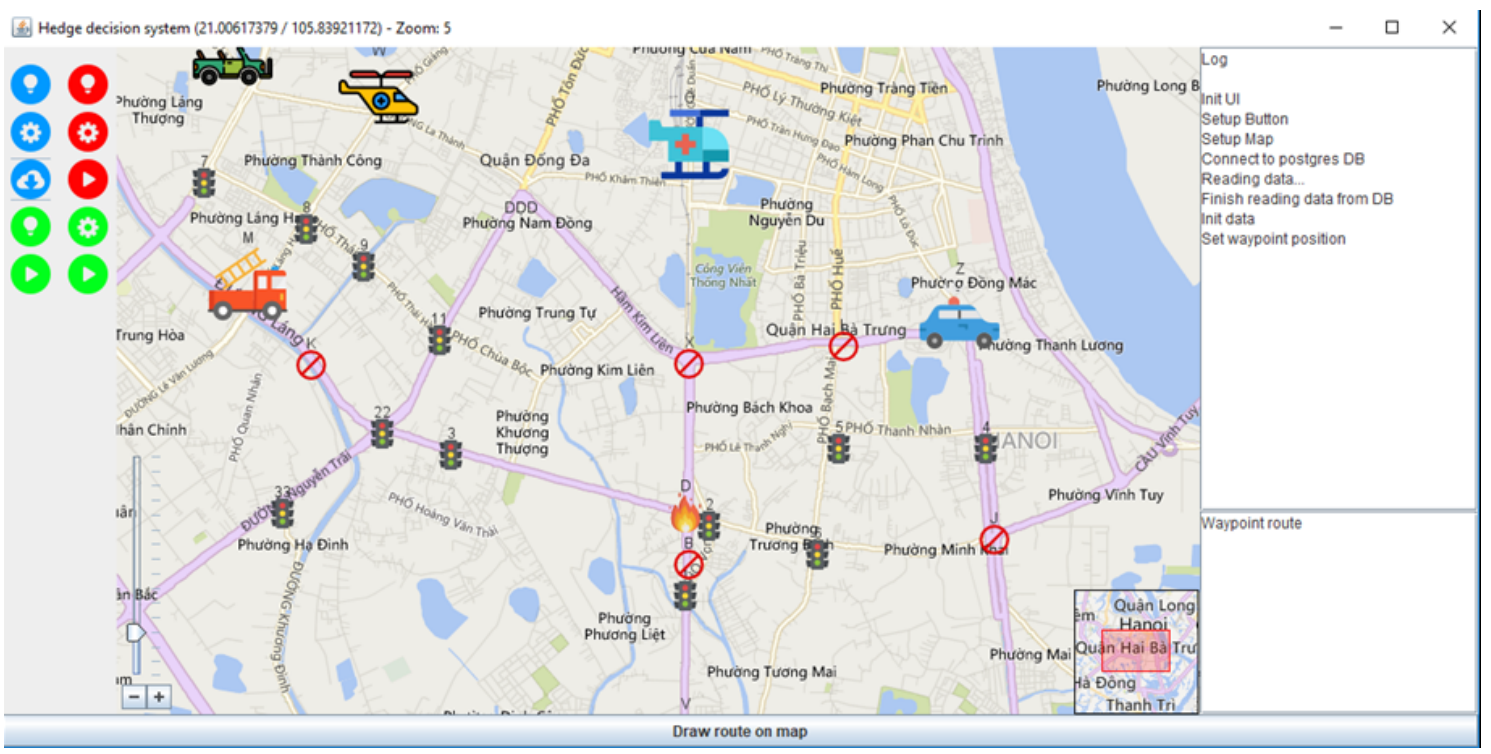

Figure 6. Initial vehicles with the proposed fire disaster appear on the Google map.

Prioritisation of service vehicles [to respond to an emergency] can enable the optimal path (including available path(s) plus the distance(s) from the current location of service vehicle(s) to the 
emergency to be identified using connected data from humans and DOE sensors is shown in Figure 7. The shortest path finding 'Dijkstra algorithm' is used to find the shortest route from the location of the service vehicle from the current location to the location of the emergency. In the event of there being multiple available service vehicles, the STC algorithm will find the distance between service vehicle(s) and the optimal service vehicle can respond to the emergency while other service vehicles will not respond. Prioritisation of service response vehicles is achieved by identifying the shortest available path(s) (route(s)) derived using connected data from humans and sensors as shown in Figure 8.

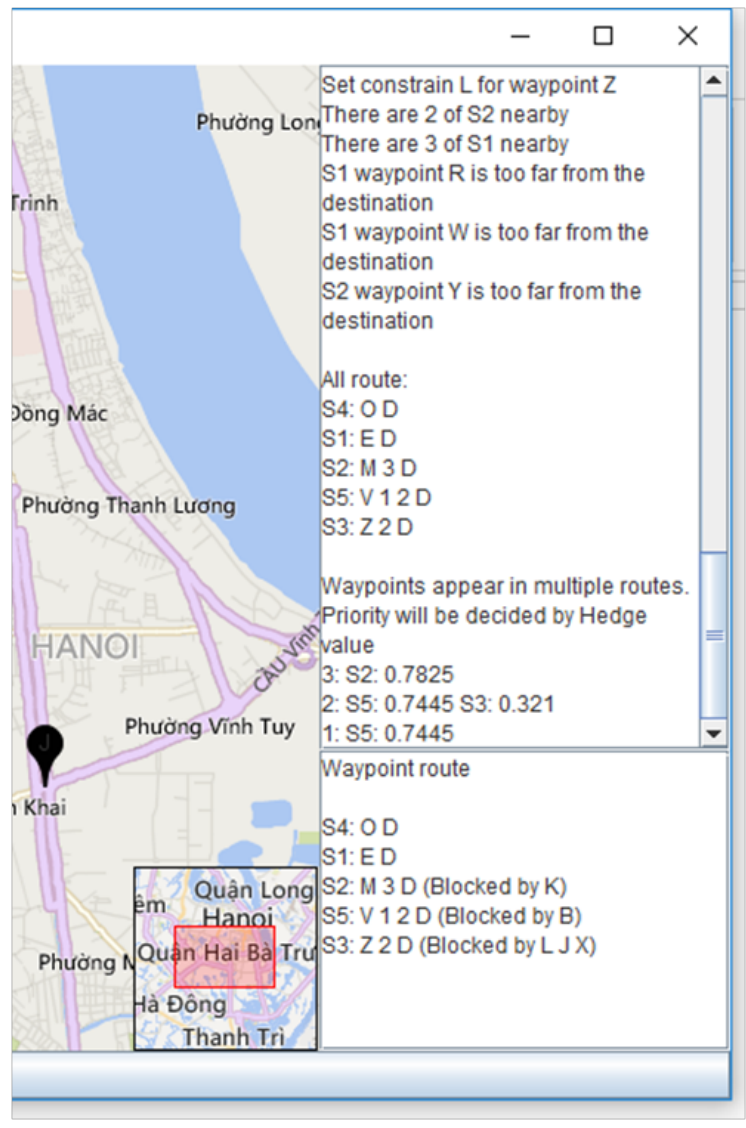

Figure 7. Priority service vehicles moved as they appear on the Google map.

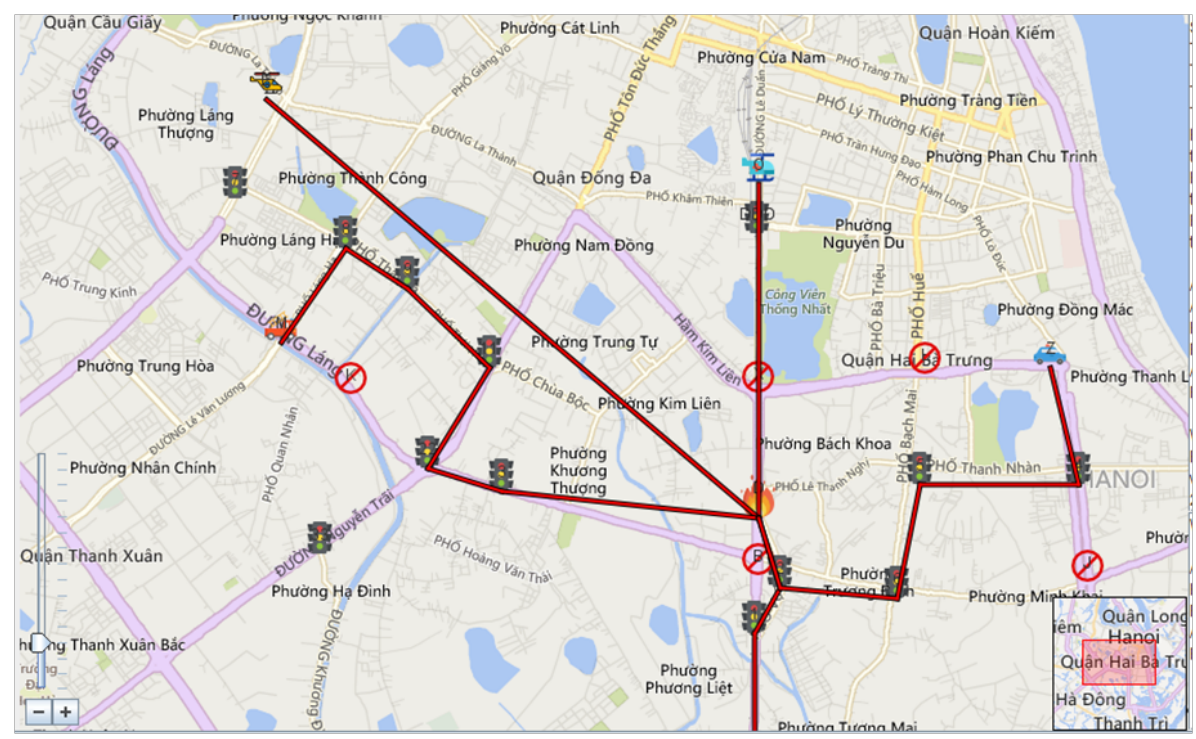

Figure 8. The route of service vehicles was appeared on the Google map. 


\subsection{Emergency Case Study in Japan}

To further evaluate the proposed approach to group decision makings using the Google maps, we conducted a further case study based on a 'real-world' disaster a decision maker can eliminate $\mathrm{SOM}$ visualisation if he has a disaster strategy in minds. He freely selects a location disaster matrix for visualisation appeared on the disaster map the available options together with sensible decisions. Most of the world's earthquakes, tsunamis, landslides, and volcanic eruptions are caused by the continuous motions in a disaster. Figure 9 illustrates the emergency location in Sendai, Japan on the Google map. Decision makers decided to adjust the map on Google for the study as shown in Figure 10 .

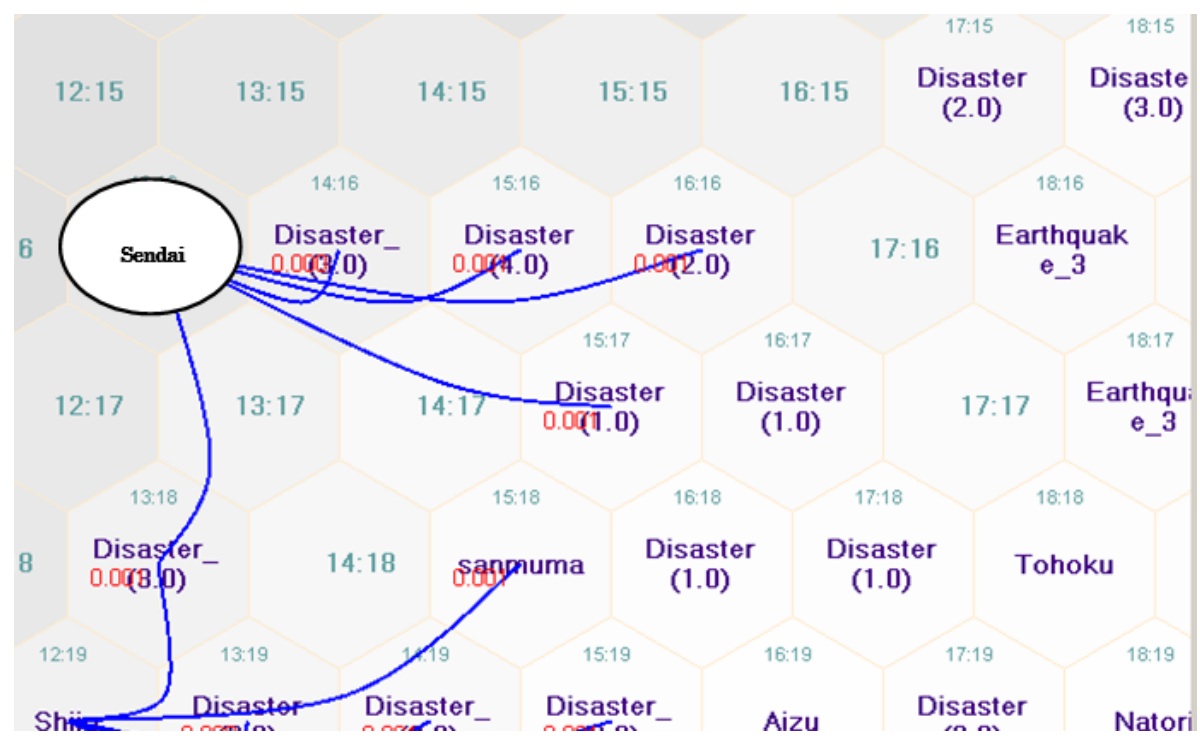

Figure 9. SOM map results in the emergency location.

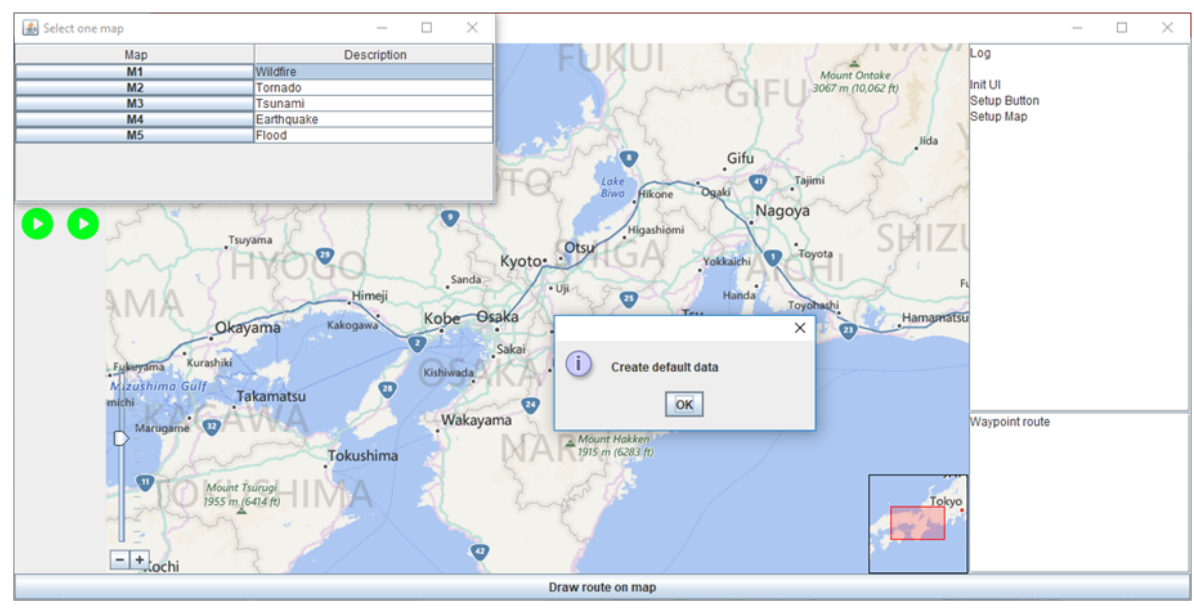

Figure 10. Identification of emergency locations in Japan on the Google map.

To ensure that comprehensive coverage [of the DOE] with all available paths identified in the emergency location(s), a decision maker can apply the STC algorithm. For example, an earthquake happened in Japan and there were emergency support vehicles available within the disaster zone (the DOE). To consider decision maker preferences in the proposed system, the experts were given in disaster strategies as objectives in group decision making to arrive at the optimal path(s) (route(s)) to expedite emergency support vehicles and provide assistance. The available options are shown in Figure 11. 
In further simulation results, we show that a decision maker can decide the shortest route to combine all emergency service vehicles to enable emergency support as shown in Figure 12. As can be seen from Figure 12, the experimental results show the location of all the emergency service vehicles in the earthquake zone. Using Hedge Algebra and Kansei evaluation based on the expert's sensibilities about disaster conditions, good decision making is promoted.

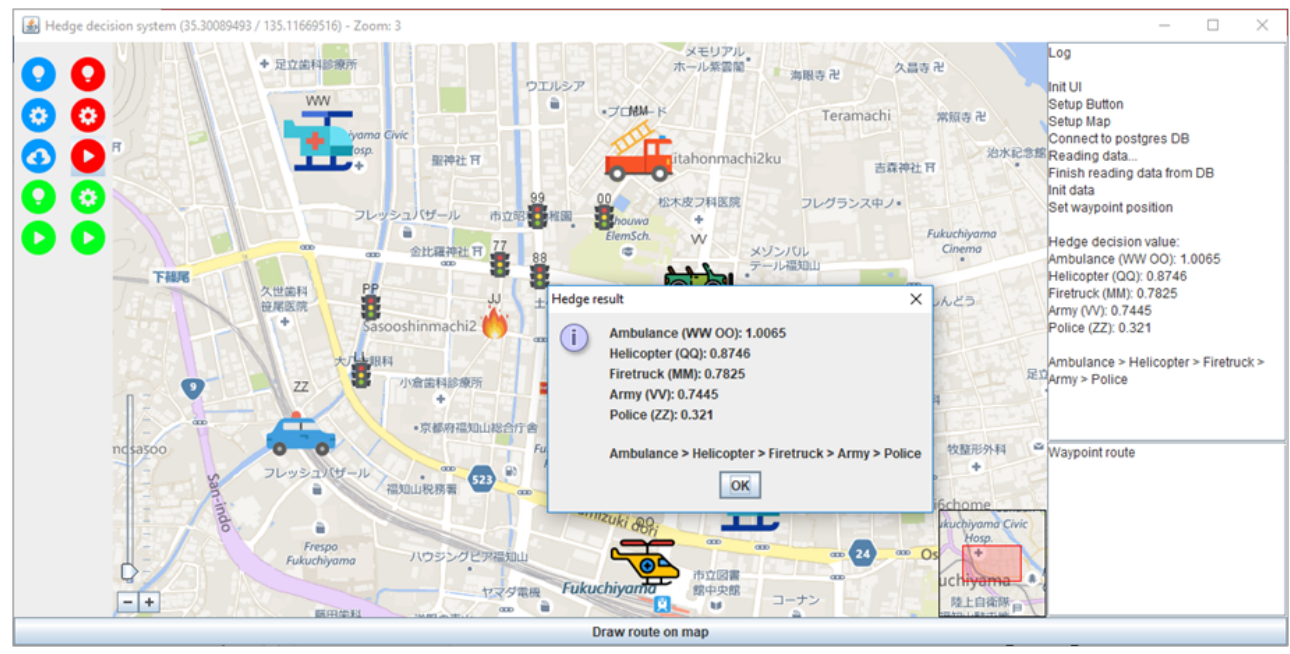

Figure 11. Priority vehicles in order to identify emergency locations in Japan on the Google map.

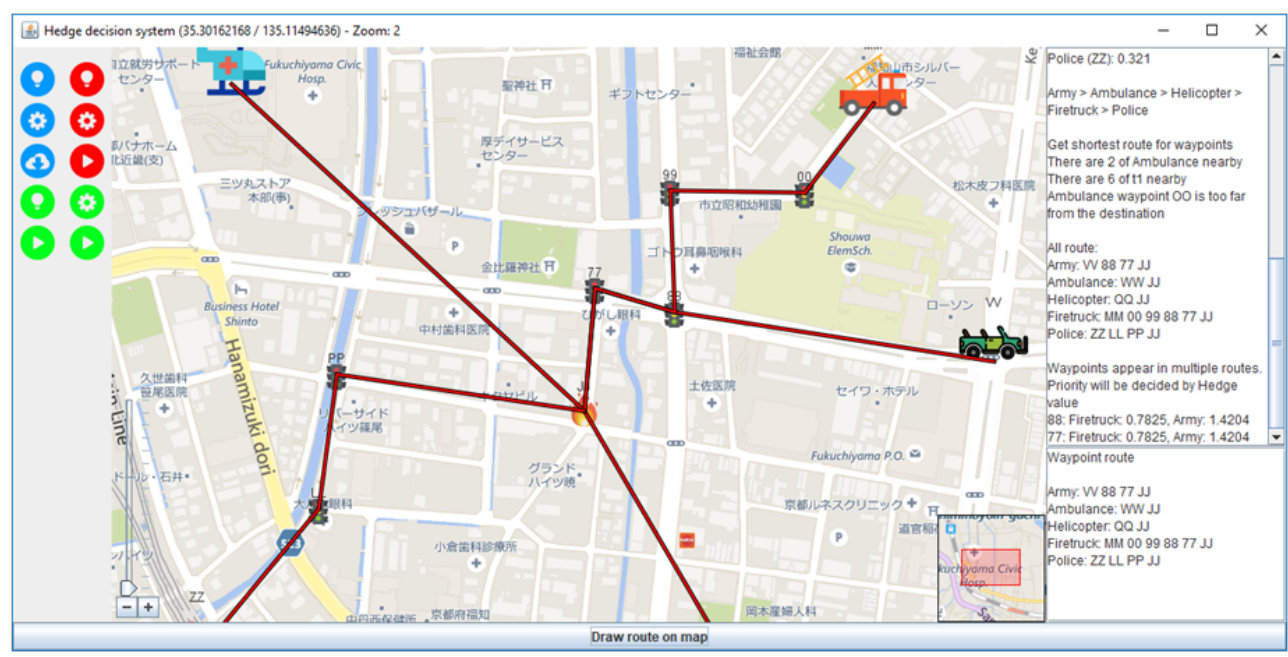

Figure 12. The route of transportation vehicles was appeared on the Google map.

\section{Results and Discussion}

In order to evaluate the effectiveness of the proposed system in simulation of disaster response, the performance of the proposed model is calculated from: (a) the average of ratings between times, and (b) the number of objectives involved in selection of the appropriate service vehicle(s) based on the available routes in the emergency zone (the DOE). In this Section we set out the methodology with results and a discussion.

To evaluate the overall performance of the proposed model with random [dynamic] obstacles (bicycles, cars, and other vehicles moving on the map (the DOE)), the performance of the proposed model was compared with a conventional method and Hed-DSS model as discussed in [41]. The proposed model, [including Hedge Algebra and Kansei evaluation] are also used to quantify decision makers' sensibilities in emergency response.

The approaches considered have performed well in evaluation and testing in the similar conditions (nodes) using the same data sets in the same map. The performance average of all cases (60 nodes) 
in simulations) was calculated for variety of cases. The proposed model far outperforms the other conventional approach in short time for the evaluation of disaster response, as shown in Figure 13.

In further evaluation and testing in simulations [the same number of 9 objectives with a variety of objectives], the experimental results show that the proposed model achieved increased computational efficiency (reduced computing time). Moreover, the results consistently show that the proposed approach in a comparative analysis [SOM integrated with the Hedge Algebra integrated Kansei evaluation approach] demonstrates a consistent improvement of response time for the proposed model results achieved, as shown in Figure 14.

Experimental results demonstrate that identifying linguistically (semantically) and evaluating the factors to be considered in a decision enables support emergency response (in an emergency zone, the DOE) to decide on the optimal routes for support and emergency service vehicles to provide an effective and efficient response. Quantifying decision maker's reasoning, perception, and linguistic (semantic) information performs a significant role in decision making under uncertain environments.

The evaluation based on experimental testing has been presented to demonstrate the efficacy of our proposed model. The results of the simulation show that the proposed model provides an effective basis for resource allocation and deployment in emergency response scenarios. Moreover, our proposed method may be used to provide decision-support in both emergency and disaster planning and in 'real-time' adaptable management of emergency response in dynamic uncertain environments.

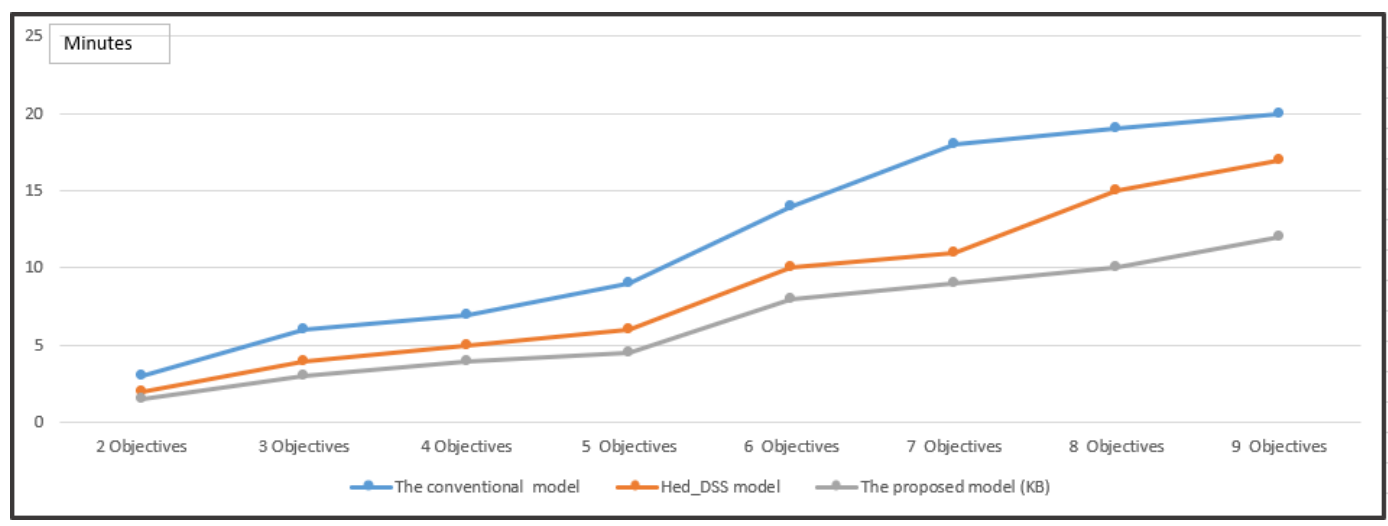

Figure 13. The Evaluation of time in 5 decision-making objects with a variety of nodes.

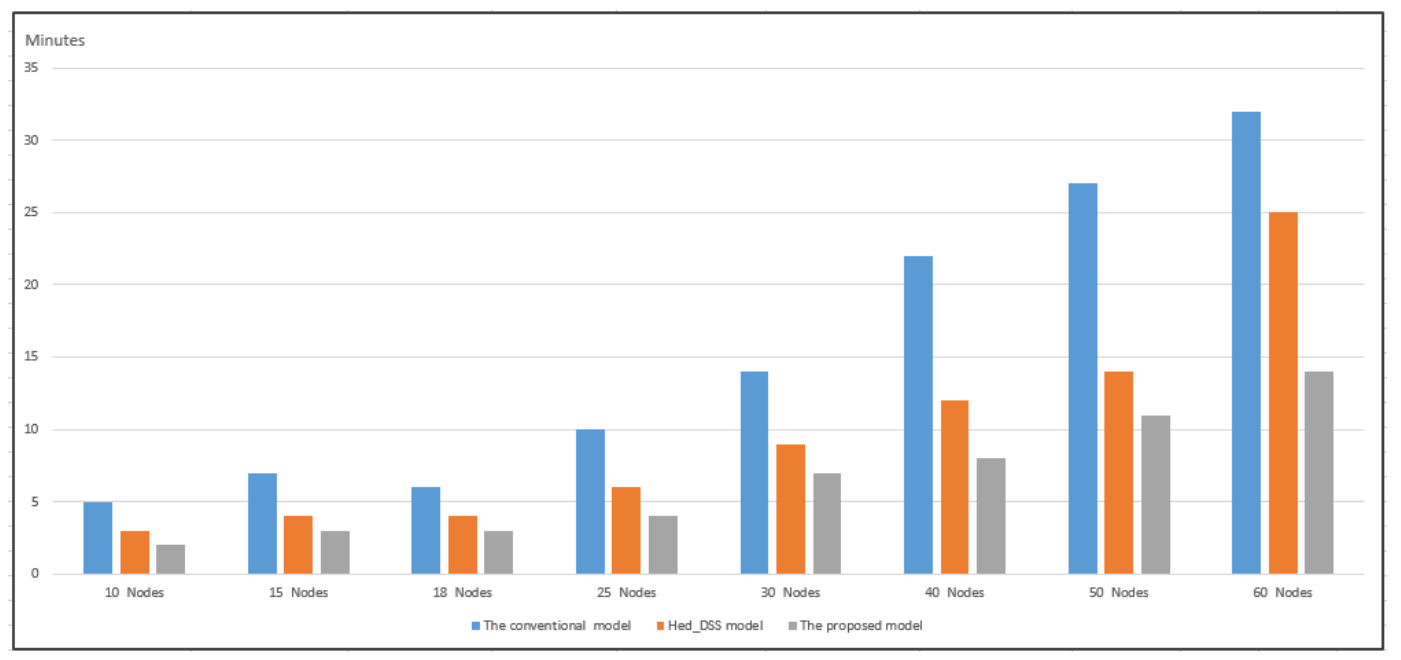

Figure 14. Evaluation of time in 5 decision-making objects with a variety of nodes.

Future Directions For Research

Every research study will resolve many questions. However, such studies will also identify 'open-research questions'. In our study such a question relates to collaborative group-based 
decision-support under dynamic uncertainty. In such group collaborative disaster recovery an interactive intelligent decision-support system is required. Investigating this question forms the basis for a future research direction.

The evaluation and experimental testing has employed a simulation which has achieved promising results. Current research is evaluating our proposed method in a 'real-world' and 'real-time' traffic management environment; Figure 15 shows a practical 'real-world' cloud-based implementation of the proposed system in a vehicle control information system with the output displayed in the in-car information system panel. The in-car application represents ongoing research with promising preliminary results; when the research is complete the results will be published in a later article.

The results of the evaluation based on the simulation and the test implementation in a 'real-world' vehicle system show that our reported results are promising and our novel approach has the potential to operate effectively in 'real-world' emergency management systems.

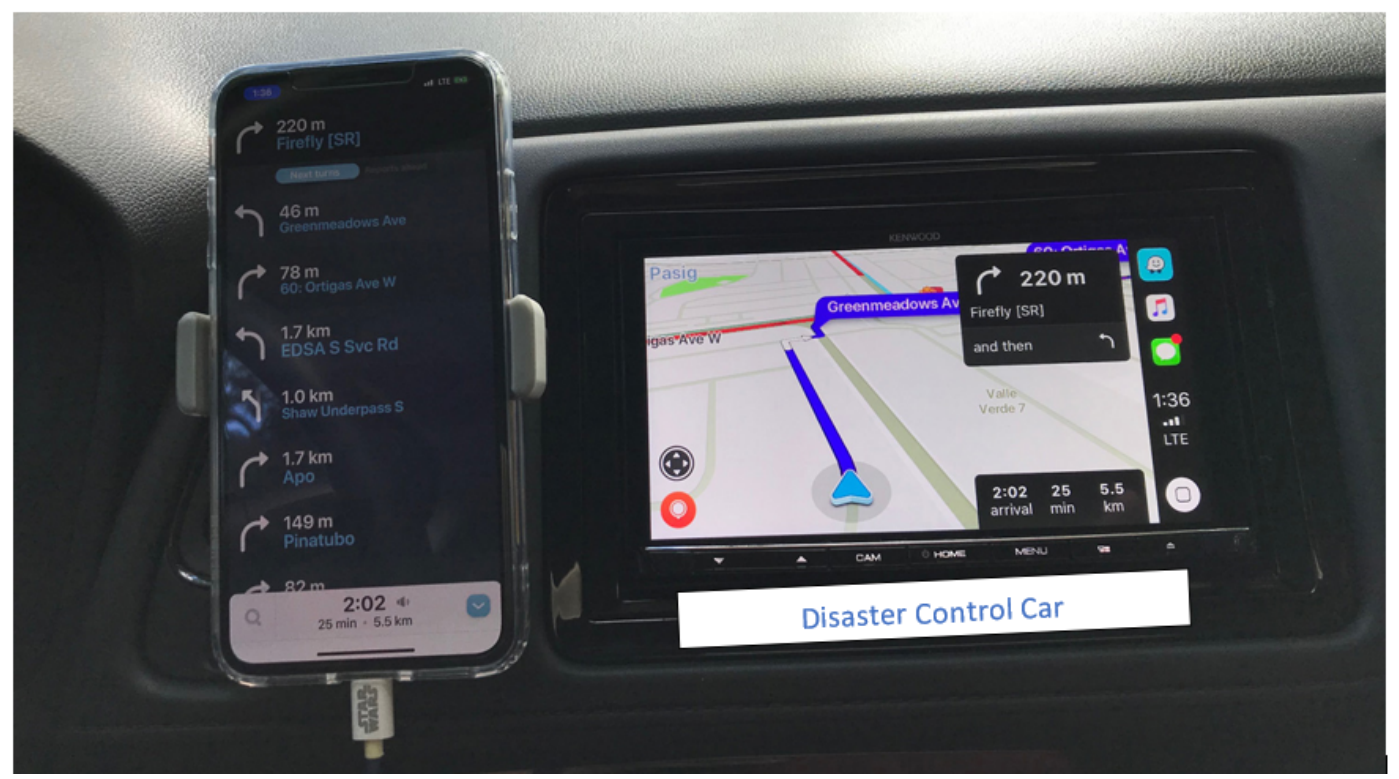

Figure 15. The demonstration of the proposed system with the output sent to a vehicle control information system panel.

\section{Concluding Observations}

In this paper we have presented a novel approach which is designed to achieve autonomous emergency emergency service vehicle control achieved by matching resources [service vehicles] with disaster strategies using group decision making in urban disaster zones.

The posited approach employs hedge algebras and Kansei evaluation to realise intelligent decision-support with improved levels of control for disaster recovery service vehicles. The proposed approach is designed to quantify qualitative factors; this method, when implemented with the modelling of human sensibilities and semantically driven reasoning and inference, has shown a significant improvement for optimal decision making in 'real-time' emergency service vehicle control in disaster recovery scenarios.

Disaster recovery forms a central function in urban planning, we posit that our novel approach to the provision of autonomous decision-support holds the prospect of improvements in disaster recovery. Moreover, we posit that our proposed method will generalise to other traffic and vehicle management scenarios in urban environments.

Author Contributions: Conceptualization, H.V.P. and P.M.; methodology, H.V.P.; software, H.V.P.; validation, H.V.P.; writing—original draft preparation, P.M. and H.V.P.; writing—review and editing, P.M.

Funding: This research is funded by Vietnam Institute for Advanced Studies in Mathematics (VIASM). 
Acknowledgments: The authors acknowledge the support provided by Hanoi University and Technology and Vietnam Institute for Advanced Studies in Mathematics (VIASM) in carrying out this research including administrative and technical support used in the experiments and evaluation.

Conflicts of Interest: The authors declare no conflict of interest.

\section{References}

1. Grove, J.M. Cities: Managing Densely Settled Social-Ecological Systems. In Principles of Ecosystem Stewardship: Resilience-Based Natural Resource Management in a Changing World; Folke, C., Kofinas, G.P., Chapin, F.S., Eds.; Springer: New York, NY, USA, 2009; pp. 281-294, doi:10.1007/978-0-387-73033-2_13.

2. Murphy, R.R. Disaster Robotics; MIT Press: Cambridge, MA, USA, 2014.

3. Pham, H.V.; Trung, N.L.; Minh, P.; Moore., P. A New Method Using Knowledge Reasoning Techniques for Improving Robot Performance in Coverage Path Planning. Int. J. Comput. Appl. Technol. 2019, 60, 57-64. [CrossRef]

4. Pham, H.V.; Moore, P.; Truong, D.X. Proposed Smooth-STC Algorithm for Enhanced Coverage Path Planning Performance in Mobile Robot Applications. Robotics 2019, 8, 44, doi:10.3390/robotics8020044. [CrossRef]

5. Van Pham, H.; Moore, P. Robot Coverage Path Planning under Uncertainty Using Knowledge Inference and Hedge Algebras. Machines 2018, 6, 46, doi:10.3390/machines6040046. [CrossRef]

6. Nguyen, C.H.; Tran, T.S.; Pham, D.P. Modeling of a semantics core of linguistic terms based on an extension of hedge algebra semantics and its application. Knowl.-Based Syst. 2014, 67, 244-262. [CrossRef]

7. Salem, B.; Nakatsu, R.; Rauterberg, M. Kansei Experience: Aesthetic, Emotions and Inner Balance. Int. J. Cogn. Inform. Nat. Intell. 2009, 3, 54-64. [CrossRef]

8. Comfort, L.K. Risk, security, and disaster management. Annu. Rev. Polit. Sci. 2005, 8, 335-356. [CrossRef]

9. Eiser, J.R.; Bostrom, A.; Burton, I.; Johnston, D.M.; McClure, J.; Paton, D.; Van Der Pligt, J.; White, M.P. Risk interpretation and action: A conceptual framework for responses to natural hazards. Int. J. Disaster Risk Reduct. 2012, 1, 5-16. [CrossRef]

10. Comfort, L.K.; Sungu, Y.; Johnson, D.; Dunn, M. Complex systems in crisis: Anticipation and resilience in dynamic environments. J. Conting. Crisis Manag. 2001, 9, 144-158. [CrossRef]

11. Kwan, M.P.; Lee, J. Emergency response after 9/11: The potential of real-time 3D GIS for quick emergency response in micro-spatial environments. Comput. Environ. Urban Syst. 2005, 29, 93-113. [CrossRef]

12. Comfort, L.K. Crisis management in hindsight: Cognition, communication, coordination, and control. Public Adm. Rev. 2007, 67, 189-197. [CrossRef]

13. Dedi. I, I.; Ghassan, B.; Biswajeet, P. Developing a decision support system for Disaster Management: Case study of an Indonesia volcano eruption. Int. J. Disaster Risk Reduct. 2018, 31, 711-721.

14. Jooho, K.; Abhijeet, D.; Makarand, H. A framework for assessing the resilience of a disaster debris management system. Int. J. Disaster Risk Reduct. 2018, 28, 674-687.

15. Khan, M.; Jamil, A.; Baik, S.W. Early fire detection using convolutional neural networks during surveillance for effective disaster management. Neurocomputing 2018, 288, 30-42.

16. Hristidis, V.; Chen, S.C.; Li, T.; Luis, S.; Deng, Y. Survey of data management and analysis in disaster situations. J. Syst. Softw. 2010, 83, 1701-1714. [CrossRef]

17. Moore, P.; Pham, H.V. On Wisdom and Rational Decision-Support in Context-Aware Systems. In Proceedings of the 2017 IEEE International Conference on Systems, Man, and Cybernetics (SMC2017), Banff, AB, Canada, 5-8 October 2017; pp. 1982-1987.

18. Moore, P.T.; Pham, H.V. On Context and the Open World Assumption. In Proceedings of the 29th IEEE International Conference on Advanced Information Networking and Applications (AINA-2015), Gwangju, Korea, 24-27 March 2015; pp. 387-392, doi:10.1109/WAINA.2015.7. [CrossRef]

19. Moore, P. Do We Understand the Relationship between Affective Computing, Emotion and Context-Awareness? Machines 2017, 5, 16, doi:10.3390/machines5030016. [CrossRef]

20. Weiser, M. The computer for the 21st century. Sci. Am. 1991, 265, 94-104. [CrossRef]

21. Endsley, M.R. Toward a theory of situation awareness in dynamic systems. Hum. Factors J. Hum. Factors Ergon. Soc. 1995, 37, 32-64, doi:10.1518/001872095779049543. [CrossRef]

22. Endsley, M.R.; Garland, D. Theoretical underpinnings of situation awareness: A critical review. Situat. Aware. Anal. Meas. 2000, 1, 24. 
23. Moore, P.; Evans, C.; Pham, H.V. Towards Integrating Emotion into Intelligent Context. In Web Information Systems Engineering-WISE 2011 and 2012 Workshops; Haller, A., Huang, G., Huang, Z., Paik, H.Y., Sheng, Q.Z., Eds.; Springer: Berlin/Heidelberg, Germany, 2013; pp. 27-40.

24. Norman, D.A. The Psychopathology of Everyday Things. In Foundations of Cognitive Psychology: Core Readings; Levitin, D.J., Ed.; The MIT Press (A Bradford Book): London, UK, 2002; Volume Part IX, doi:10.1016/B978-0-08-051574-8.50006-6.

25. Kokar, M.M.; Endsley, M.R. Situation Awareness and Cognitive Modeling. IEEE Intell. Syst. 2012, 27, 91-96. doi:10.1109/MIS.2012.61. [CrossRef]

26. Moore, P.; Thomas, A.; Tadros, G.; Barolli, L.; Pham, H.V. Situation Awareness for Enhanced Patient Management. In Proceedings of the 7th International Conference on Complex, Intelligent, and Software Intensive Systems (CISIS 2012), The Third International Workshop on Intelligent Context-Aware Systems (ICAS 2013), Taichung, Taiwan, 3-5 July 2013; pp. 493-498, doi:10.1109/CISIS.2013.90. [CrossRef]

27. Moore, P.; Pham, H.V. Personalization and rule strategies in human-centric data intensive intelligent context-aware systems. Knowl. Eng. Rev. 2015, 30, 140-156, doi:10.1017/S0269888914000265. [CrossRef]

28. Checkland, P.; Holwell, F. Information, Systems and Information Systems: Making Sense of the Field; John Wiley and Sons: Chichester, UK, 1998.

29. Deakin, M.; Waer, H.A. From intelligent to smart cities. Intell. Build. Int. 2011, 3, 140-152, doi:10.1080/17508975.2011.586671. [CrossRef]

30. Thomas, A.M.; Moore, P.; Shah, H.; Evans, C.; Sharma, M.; Xhafa, F.; Mount, S.; Pham, H.V.; Wilcox, A.J.; Patel, A.; et al. Smart care spaces: Needs for intelligent at-home care. Int. J. Space-Based Situat. Comput. 2013, 3, 35-44, doi:10.1504/IJSSC.2013.051988. [CrossRef]

31. Thomas, A.; Moore, P.; Evans, C.; Shah, H.; Sharma, M.; Mount, S.; Xhafa, F.; Pham, H.; Barolli, L.; Patel, A.; et al. Smart care spaces: Pervasive sensing technologies for at-home care. Int. J. Ad Hoc Ubiquitous Comput. 2014, 16, 268-282, doi:10.1504/IJAHUC.2014.064862. [CrossRef]

32. Cisco. Fog Computing and the Internet of Things: Extend the Cloud to Where the Things Are; Cisco: San Jose, CA, USA, 2017.

33. How Fog Computing May Power the Healthcare Internet of Things. Available online: https: / / healthitanalytics.com/features/how-fog-computing-may-power-the-healthcare-internet-of-things (accessed on 1 August 2019).

34. Pallis, G. Cloud Computing: The New Frontier of Internet Computing. IEEE Internet Comput. 2010, 14, 70-73, doi:10.1109/MIC.2010.113. [CrossRef]

35. Moore, P.; Van Pham, H. A Fog Computing Model for Pervasive Connected Healthcare in Smart Environments. Int. J. Grid Util. Comput. 2019, 10, 375-391.

36. Chen, S.; Liu, J.; Wang, H.; Xu, Y.; Augusto, J.C. A linguistic multi-criteria decision making approach based on logical reasoning. Inf. Sci. 2014, 258, 266-276. [CrossRef]

37. Liu, J.; Li, W.; Chen, S.; Xu, Y. An axiomatizable logical foundation for lattice-ordered qualitative linguistic approach for reasoning with words. Inf. Sci. 2014, 263, 110-125. [CrossRef]

38. Moore, P.; Pham, H.V. Intelligent Context with Decision Support under Uncertainty. In Proceedings of the 6th International Conference on Complex, Intelligent, and Software Intensive Systems (CISIS 2012), Palermo, Italy, 4-6 July 2012; pp. 977-982, doi:10.1109/CISIS.2012.17. [CrossRef]

39. Moore, P.; Pham, H.V. Towards Implementing Emotion into Intelligent Context. In Web Information Systems Engineering (WISE 2012), The International Workshop on Advanced Reasoning Technology for e-Science (ART2012); Springer: Berlin, Germany, 2012; Volume LNCS 7652, pp. 27-40.

40. Kohonen, T. The self-organizing map. Proc. IEEE 1990, 78, 1464-1480, doi:10.1109/5.58325. [CrossRef]

41. Pham, H.V.; Moore, P.; Thuan, D.P. A new Approach using Hedge Algebra combined with Kansei Evaluation for Intelligent Disaster Decision Support and its Application. In Frontiers in Intelligent Computing: Theory and Applications, Proceedings of the 7th International Conference on FICTA (2018), Volume 2; Satapathy, S.C., Bhateja, V., Nguyen, B.L., Nguyen, G.N., Le, D.N., Eds.; Springer: Singapore, 2019; Volume XII, p. 347, in press. [CrossRef]

(C) 2019 by the authors. Licensee MDPI, Basel, Switzerland. This article is an open access article distributed under the terms and conditions of the Creative Commons Attribution (CC BY) license (http://creativecommons.org/licenses/by/4.0/). 